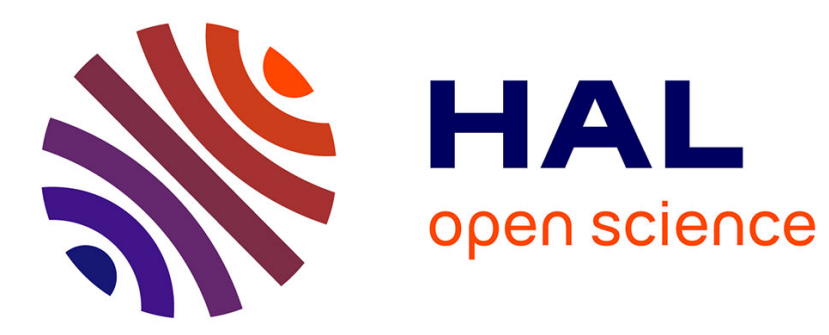

\title{
Strange attractor identification and state observation under sparse measurements
}

Yassine Khaled, Jean-Pierre Barbot, Djamila Benmerzouk, Krishna Busawon, Ghanes Malek

\section{- To cite this version:}

Yassine Khaled, Jean-Pierre Barbot, Djamila Benmerzouk, Krishna Busawon, Ghanes Malek. Strange attractor identification and state observation under sparse measurements. Environment-Friendly Energies and Applications, Jun 2012, Newcastle, upon tyne, United Kingdom. hal-00749736

\section{HAL Id: hal-00749736 \\ https://hal.inria.fr/hal-00749736}

Submitted on 8 Nov 2012

HAL is a multi-disciplinary open access archive for the deposit and dissemination of scientific research documents, whether they are published or not. The documents may come from teaching and research institutions in France or abroad, or from public or private research centers.
L'archive ouverte pluridisciplinaire HAL, est destinée au dépôt et à la diffusion de documents scientifiques de niveau recherche, publiés ou non, émanant des établissements d'enseignement et de recherche français ou étrangers, des laboratoires publics ou privés. 


\title{
Strange attractor identification and state observation under sparse measurements
}

\author{
Y. Khaled ${ }^{1,2}$, J-P. Barbot ${ }^{1,3}$, D. Benmerzouk ${ }^{2}$, K. Busawon ${ }^{4}$ and M. Ghanes ${ }^{1}$ \\ ${ }^{1}$ ECS-Lab, ENSEA, 6 Avenue du Ponceau, 95014 Cergy-Pontoise, France. \\ 2 Department of Mathematics, Tlemcen University, Algeria. \\ ${ }^{3}$ EPI Non-A, INRIA. \\ ${ }^{4}$ School of Computing, Engineering and Information Sciences, \\ Northumbria University, Newcastle upon Tyne NE1 8ST, U.K.
}

\begin{abstract}
In this paper, a new method of strange attractor identification, under sparse measurement, is proposed this method is based on the concept of compressive sensing. For this, some particular impulsive observers have been presented and adding a decision scheme linked to diagnosis method, the identification of the strange attractor and state observation are done. Some simulations results are given in order to highlight the well founded of the proposed design.
\end{abstract}

Index Terms - Impulsive observer; synchronization under sampling; Compressive Sensing; Chaos.

\section{INTRODUCTION}

More and more in control theory, the sparsity property is invoked and this in many contexts: in sensors placement [20], in linear optimal control [4].

This growing up interest for sparsity properties comes from signal processing theory where compressing sensing method allows to recover a signal even if the signal sampling frequency is smaller than the Nyquist-Shannon sampling frequency (undersampling). For more details on the topic of undersampling, the reader is invited to refer, for instance, to the interesting work on compressive sensing in [8] and [5]. In effect, Compressive Sensing (CS) is based on the assumptions that the signal is located in a former transmitted space expanded by some appropriate basis [10] and that the so-called CS matrix verifies the well-known Restricted Isometries Property (RIP) [5]. Under such assumptions, the signal can be reconstructed by using the so called sparse regularized linear regression techniques.

This result naturally leads one to ask whether or not it is possible to bypass the Nyquist-Shannon sampling frequency constraint in control system theory and this for many purposes; a closed loop control scheme, diagnosis, active fault tolerant control, real time decision,... This question, in turn, generates several essential questions:

i) How can we translate CS from a signal context to a system context?

ii) What is the appropriate basis in a system context?

iii) How do we verify the RIP condition in a system context?

iv) How can we bypass the optimization algorithm in order to deal with real time algorithms?
An answer to question i) is partially given in the signal processing literature, such as in [21], whereby a model-based CS is presented; even though such kind of model is quite different from usual dynamical models.

Answers to questions ii) is implicitly given in several papers on observation or diagnosis of systems [1], [2], [3]. Specifically, one appropriate basis is the basis of normal form [12], [17] flow associated to Whitney topologies with all restrictive considerations with respect to the parameter genericity and sensibility [9]. In this paper, an answer to question ii) is given with respect to a class of chaotic systems. It is important, to mention that in this paper it is assumed that only one basis element is assumed different from zero and the case of multi basis elements different from zero, clustered or not, will be treated in future paper.

The answer to question iii) will be treated in an upcoming paper for the class of linear system. Nevertheless, if we consider the observability normal form [22], only a subset of the normal form verifies the RIP. For instance, it is obvious that a subset of the unobservable system does not verify the RIP.

An answer to the question iv) is proposed in [13] and will be use here for illustrated the answer to question ii). More precisely, an impulsive observer, which bypass the optimization algorithm under specific conditions, will be presented in the present paper for the first time in nonlinear context and coupled to a proposed identification algorithm.

The paper is organized as follow: in the next section some stability results for a class of impulsive systems are recalled, in section three some new impulsive observers are recalled [13], in section four a multi impulsive observers design is presented on the basis of classical multi observer design [1]. Finally, we use the proposed method on the strange attractor identification and we give an example with simulation in order to highlight the well founded of our approach and the paper and with a conclusion and perspectives.

\section{Some STABILITY RESUlTS FOR A CLASS OF IMPULSIVE SYSTEM}

In this section we first recall some stability definitions, for more details see [6], [13]. Let us consider the following 
dynamic:

$$
\dot{x}(t)=f(x(t))
$$

where $t>0, x(t) \in \mathbb{R}^{n}, f: \mathbb{R}^{n} \rightarrow \mathbb{R}^{n}$.

Definition 1: (A of a ball)

The ball $B_{\varepsilon}=\left\{x \in \mathbb{R}^{n} /\|X\|<\varepsilon\right\}$ is attractive on $B_{\rho}=\left\{x \in \mathbb{R}^{n} /\|X\|<\rho\right\}$ for the dynamic (1) if there exists a class $\mathcal{L}^{1}$ function $\beta$ such that $\forall x(0):=x_{0} \in B_{\rho}$ the flow $\phi\left(t, x_{O}\right)$ of (1) verifies $\forall t>0\left\|\phi\left(t, x_{O}\right)\right\|_{\varepsilon} \leq \beta(t)$.

Definition 2: (AS of a ball)

The ball $B_{\varepsilon}=\left\{x \in \mathbb{R}^{n} /\|X\|<\varepsilon\right\}$ is asymptotically stable on $B_{\rho}=\left\{x \in \mathbb{R}^{n} /\|X\|<\rho\right\}$ for the dynamic (1) if it is both stable and attractive on $B_{\rho}$.

Definition 3: GAS of a ball The ball $B_{\varepsilon}=\{x \in$ $\left.\mathbb{R}^{n} /\|X\|<\varepsilon\right\}$ is globally asymptotically stable for the dynamic (1) if it asymptotically stable on $\mathbb{R}^{n}$.

Through the paper we will consider the following class of impulsive dynamics:

$$
\left\{\begin{array}{l}
\dot{x}_{1}(t)=f_{1}\left(x_{1}(t), x_{2}(t), t\right) ; \quad t \neq t_{k} \\
\dot{x}_{2}(t)=f_{2}\left(x_{1}(t), x_{2}(t), t\right) ; \quad t \neq t_{k} \\
x_{1}\left(t_{k}^{+}\right)=0 \\
x_{2}\left(t_{k}^{+}\right)=x_{2}\left(t_{k}\right)
\end{array}\right.
$$

where $x_{1}(t) \in \mathbb{R}^{p}, x_{2}(t) \in \mathbb{R}^{n-p}, f_{1}: \mathbb{R}^{n} \rightarrow \mathbb{R}^{p}$ and $f_{2}: \mathbb{R}^{n} \rightarrow \mathbb{R}^{n-p}$.

The resiting sequence $t_{k} \in T=\left\{t_{i}: i \in \mathbb{N}\right\} \subset \mathbb{R}^{+}$verifies that there exists $\tau_{\min }$ and $\tau_{\max }$ with $0<\tau_{\min }<\tau_{\max }$ such that:

$$
\forall i>0: t_{i+1} \geq t_{i}+\tau_{\min } \quad \text { and } \quad t_{i+1} \leq t_{i}+\tau_{\max }
$$

Additionally, we define:

$$
\begin{gathered}
\theta_{k} \triangleq t_{k+1}-t_{k} \\
x\left(t_{k}^{+}\right) \triangleq \lim _{h \rightarrow 0^{+}} x\left(t_{k}+h\right) \text { and } x\left(t_{k}^{-}\right) \triangleq \lim _{h \rightarrow 0^{+}} x\left(t_{k}-h\right) \triangleq x\left(t_{k}\right)
\end{gathered}
$$

In order to avoid Zenon phenomena, we assume that:

$$
t_{i}+\tau_{\min }<t_{i+1} \quad \text { and } \quad t_{i}+\tau_{\max }>t_{i+1}
$$

From the definition of global asymptotical stability of a ball $B_{\varepsilon}$, and considering first a specific instant just after it is possible first to exhibit some sufficient stability conditions for the system (2). Nevertheless, for this we must set some assumptions:

Assumption 1: $f_{1}$ is at least uniformly locally Lipschitz, where $l_{1}$ and $l_{2}$ are respectively the Lipschitz constants with respect to $x_{1}$ and $x_{2}$.

Assumption 2: $f_{2}\left(x_{2}, x_{1}, t\right)=A x_{2}+B(t) x_{1}$ with $\forall t \geq 0$ $B(t)<M$.

Theorem 1: If the system (2) verifies assumptions 1, 2 and there exists a strictly positive definite function $V_{2}: \mathbb{R}^{n-p} \mapsto \mathbb{R}^{+} V_{2} \in \mathcal{C}^{1}$, which satisfies the following conditions:

1) $\forall x_{2} \neq\left. 0 \frac{\partial V_{2}(\cdot)}{\partial x_{2}}\right|_{x(2)} A x_{2}<-l_{v}\left\|x_{2}\right\|_{2}$

\footnotetext{
${ }^{1}$ decreasing continuous function from $\mathbb{R}^{+}$to $\mathbb{R}^{+}$, with $\beta(\infty)=0$
}

2) $\left.\frac{\partial V_{2}(\cdot)}{\partial x_{2}}\right|_{x(2)}$ is Lipschitz where $k_{v}$ is the Lipschitz constant.

then, there exists a $\theta_{\max }>0$ such that for all sampling sequence $\theta_{k} \leq \theta_{\max }, x_{2}\left(t_{K}\right)$ converge globally asymptotically to zero for $k \rightarrow+\infty$.

Proof 1: Starting with $V_{2}\left(x_{2}(t)\right)$ for $t \in\left[t_{k}, t_{t+k}\right.$, we get:

$$
\dot{V}_{2}\left(x_{2}(t)\right)=\left.\frac{\partial V_{2}(.)}{\partial x_{2}}\right|_{x_{2}(.)} A x_{2}+\left.\frac{\partial V_{2}(.)}{\partial x_{2}}\right|_{x_{2}(.)} B(t) x_{1}
$$

so from assumption 2 and condition $2, \forall t \in\left[t_{k}, t_{t+k}\right.$ [, we have:

$$
\dot{V}_{2}\left(x_{2}(t)\right) \leq-l_{v}\left\|x_{2}\right\|_{2}^{2}+k_{v} M\left\|x_{1}\right\|_{2}\left\|x_{2}\right\|_{2}
$$

As $x_{1}$ is reseted to zero at each impulsive instant and $f_{1}$ is Lipschitz for all $t \in\left[t_{k}, t_{t+k}\left[, x_{1}(t)<x_{1, \max }(k)\left(x_{1, \max }(k)\right.\right.\right.$ will be define in the next), we obtain:

$$
\dot{V}_{2}\left(x_{2}(t)\right) \leq-l_{v}\left\|x_{2}\right\|_{2}^{2}+k_{v} M\left\|x_{1, \max }(k)\right\|_{2}\left\|x_{2}\right\|_{2}
$$

So $\dot{V}_{2}\left(x_{2}(t)\right) \leq 0$ if

$\left\|x_{2}(t)\right\|_{2}>\frac{k_{v} M\left\|x_{1, \max }(k)\right\|_{1}}{l_{v}}:=\varsigma(k) \geq \frac{k_{v} M\left\|x_{1, \max }(k)\right\|_{2}}{l_{v}}$

So we conclude that the ball $B_{\varsigma}=\left\{x_{2} \in \mathbb{R}^{n-p} /\left\|x_{2}\right\|_{2} \leq \varsigma\right\}$ is globally asymptotically stable. Nevertheless, $\varsigma$ is function of $k$ and as we will see hereafter, also function of $\left\|x_{2}\left(t_{k}\right)\right\|_{2}$, consequently we have to investigate the behavior of $\varsigma$ with respect to $k$. For this purpose, let us define the Lyapunov functions $V_{1}\left(x_{1}(t)\right)$ as:

$$
V_{1}\left(x_{1}(t)\right)=\left\|x_{1}(t)\right\|_{1}=\sum_{i=1}^{p}\left|x_{i}(t)\right|
$$

then, the derivative of function $V_{1}\left(x_{1}(t)\right)$ is given by:

$$
\dot{V}_{1}\left(x_{1}(t)\right)=\sum_{i=1}^{p} \operatorname{sign}\left(x_{i}(t)\right) \frac{\partial x_{i}(t)}{\partial t}
$$

The derivative of $V_{1}$ is a multivalued function and so some problems may occur for $x_{i}(t)=0$ because $\operatorname{sign}(0) \in[-1,1]$. But here the worth case is always considered so everywhere $\operatorname{sign}\left(x_{i}(t)\right) \frac{\partial x_{i}(t)}{\partial t}$ is majored by $\left|\frac{\partial x_{i}(t)}{\partial t}\right|$. Consequently, we have

$$
\dot{V}_{1}\left(x_{1}(t)\right) \leq \sum_{i=1}^{p}\left|\dot{x}_{i}(t)\right|=\left\|f_{1}(x)\right\|_{1}
$$

Since from assumption $1, f_{1}$ is Lipschitz, then there exists $l_{1}>$ 0 (Lipschitz constant with respect $x_{1}$ ) and $l_{2}>0$ (Lipschitz constant with respect $x_{2}$ ) such that

$$
\begin{aligned}
\dot{V}_{1}\left(x_{1}(t)\right) & \leq l_{1}\left\|x_{1}(t)\right\|_{1}+l_{2}\left\|x_{2}(t)\right\|_{1} \\
& =l_{1} V_{1}\left(x_{1}(t)\right)+l_{2}\left\|x_{2}(t)\right\|_{1}
\end{aligned}
$$

which implies as $V_{1}\left(t_{k}^{+}\right)=0$, we have $\forall t \in\left[t_{k}, t_{t+k}[\right.$ :

$$
V_{1}\left(x_{1}(t)\right) \leq l_{2} \int_{t_{k}}^{t} e^{l_{1}(t-\tau)}\left\|x_{2}(t)\right\|_{1} d \tau
$$


this implies that

$$
V_{1}\left(x_{1}\left(t_{k+1}\right)\right) \leq \frac{l_{2}}{l_{1}}\left(e^{l_{1}\left(t_{k+1}-t_{k}\right)}-1\right)\left\|x_{2}\left(t_{k}\right)\right\|_{1}
$$

From (6) it is possible to compute $\left\|x_{1 \max }(k)\right\|_{1}$

$$
\left\|x_{1 \max }(k)\right\|_{1}=\frac{l_{2}}{l_{1}}\left(e^{l_{1}\left(t_{k+1}-t_{k}\right)}-1\right)\left\|x_{2}\left(t_{k}\right)\right\|_{1}
$$

and therefore using (7) in (4), we obtain the following inequality

$$
\left\|x_{2}(t)\right\|_{2}>\frac{k_{v} M}{l_{v}} \frac{l_{2}}{l_{1}}\left(e^{l_{1}\left(t_{k+1}-t_{k}\right)}-1\right)\left\|x_{2}\left(t_{k}\right)\right\|_{1}
$$

If $\dot{V}_{2}<0$ then $\left\|x_{2}(t)\right\|_{2} \geq k_{v}\left\|x_{2}\left(t_{k+1}\right)\right\|_{2} \forall t \in\left[t_{k}, t_{k+1}\right]$, and with respect to the norm relation we get

$$
\left\|x_{2}\left(t_{k+1}\right)\right\|_{2}>\left.\frac{k_{v} M}{l_{v}} \frac{l_{2}}{l_{1}}\left(e^{l_{1}\left(t_{k+1}-t_{k}\right)}-1\right) \sqrt{n-p}\left\|x_{2}\left(t_{k}\right)\right\|\right|_{2}
$$

and we want $\left\|x_{2}\left(t_{k+1}\right)\right\|_{2}<\left\|x_{2}\left(t_{k}\right)\right\|_{2}$ in order to obtain a contracting sequence, this leads to the following inequality

$$
\left\|x_{2}\left(t_{k}\right)\right\|_{2}>\left.\frac{k_{v} M}{l_{v}} \frac{l_{2}}{l_{1}}\left(e^{l_{1}\left(t_{k+1}-t_{k}\right)}-1\right) \sqrt{n-p}\left\|x_{2}\left(t_{k}\right)\right\|\right|_{2}
$$

Which verified if

$$
\frac{k_{v} M}{l_{v}} \frac{l_{2}}{l_{1}}\left(e^{l_{1}\left(t_{k+1}-t_{k}\right)}-1\right) \sqrt{n-p}<1
$$

so if all $t_{k+1}-t_{k}=\theta_{k} \leq \theta_{\max }$ with

$$
\theta_{\max }=\frac{1}{l_{1}} \log \left(\frac{l_{v} l_{1}}{k_{v} M l_{2} \sqrt{n-p}}+1\right)
$$

the sequence $x_{2}\left(t_{k}\right)$ converges to zero for $k \rightarrow \infty$.

Corollary 1: Assume that the conditions and assumptions of the theorem 1 hold for system (2), then $x_{1}(t)$ and $x_{2}(t)$, converge to zero for $t \rightarrow+\infty$.

Proof 2: From the proof of theorem 1 it is guarantee that $x_{1}(t) \leq\left\|x_{1 \max }(k)\right\|_{1}=\frac{l_{2}}{l_{1}}\left(e^{l_{1}\left(t_{k+1}-t_{k}\right)}-1\right)\left\|x_{2}\left(t_{k}\right)\right\|_{1}$ $\forall t \in\left[t_{k}, t_{k+1}\right]$ and as $\left\|x_{2}\left(t_{k}\right)\right\|_{1}$ converge to zero for $k \rightarrow$ $+\infty$ then $\left\|x_{1}(t)\right\|_{1} \rightarrow 0$ for $t \rightarrow+\infty$.

Now from the fact that $\left\|x_{2}(t)\right\|_{2} \leq k_{v}\left\|x_{2}\left(t_{k}\right)\right\|_{2}$

$\forall t \in\left[t_{k}, t_{k+1}\right]$ and $\left\|x_{2}\left(t_{k}\right)\right\|_{2} \rightarrow 0$ for $k \rightarrow+\infty$ we conclude that $\left\|x_{2}(t)\right\|_{2} \rightarrow 0$ for $t \rightarrow+\infty$.

\section{IMPULSIVE OBSERVER DESIGN}

Now, we consider the following class of systems:

$$
\left\{\begin{array}{l}
\dot{x_{1}}(t)=f_{1}\left(x_{1}, x_{2}\right) \\
\dot{x_{2}}(t)=f_{2}\left(x_{1}, x_{2}\right) \\
y\left(t_{k}\right)=x_{1}\left(t_{k}\right)
\end{array}\right.
$$

Where $x(t)=\left(x_{1}(t)^{T}, x_{2}(t)^{T}\right)^{T} \in \mathbb{R}^{n}$ is the state, with $x_{1}(t) \in \mathbb{R}^{p}, x_{2}(t) \in \mathbb{R}^{n-p}$ and $y\left(t_{k}\right) \in \mathbb{R}^{p}$ is the output. The functions $f_{1}$ and $f_{2}$ are $\mathcal{C}^{1}$ and Lipschitz. Moreover the state dynamic evolve in a bounded space.

The Impulsive observer, for system (10) has the following form

$$
\left\{\begin{array}{l}
\dot{\hat{x}}_{1}(t)=f_{1}\left(\hat{x}_{1}(t), \hat{x}_{2}(t)\right) \\
\dot{\hat{x}}_{2}(t)=f_{2}\left(\hat{x}_{1}(t), \hat{x}_{2}(t)\right) \\
\hat{x}_{1}\left(t_{k}^{+}\right)=x_{1}\left(t_{k}\right)
\end{array}\right.
$$

From (11) and (10), we can set the dynamic of the observation errors $\left(e_{1}:=x_{1}-\hat{x}_{1}\right.$ and $\left.e_{2}:=x_{2}-\hat{x}_{2}\right)$ as follow:

$$
\left\{\begin{array}{l}
\dot{e}_{1}(t)=f_{1}\left(x_{1}(t), x_{2}(t)\right)-f_{1}\left(\hat{x}_{1}(t), \hat{x}_{2}(t)\right) \\
\dot{e}_{2}(t)=f_{2}\left(x_{1}(t), x_{2}(t)\right)-f_{2}\left(\hat{x}_{1}(t), \hat{x}_{2}(t)\right) \\
e_{1}\left(t_{k}^{+}\right)=0
\end{array}\right.
$$

Now, we can give an obvious corollary to the previous theorem:

Corollary 2: If the system (12) verifies the assumptions 1, 2 and conditions of theorem 1 then there exists $\theta_{\max }$ such that for all impulsive sequence which verify $\forall k \in \mathbb{N} \theta_{k} \leq \theta_{\max }$ the state of the impulsive observer (11) converge to the state of the system (10).

\section{Multi OBSERVERS DESIGN}

Usually the choice of basis in compressive sensing is an essential task [18] (curvelet, wavelet,..) in signal processing. Obviously some knowledge on signal to recover are very useful for design a decompression algorithm. So, in control theory, one of the main question in order to develop a method inspired by compressive sensing technique is, what is the appropriate base? Starting from the work of Poincar [16] on normal form for stability study and extended to control theory by W. Kang and A. Krener [11], [12] in order to analyze the system controllability. Moreover, some authors have used normal forms for the study of observability property [14], [19], [22], from these works, it is natural to investigate if observability normal forms can be an appropriate base for state observation and system detection under sparse measurement. Nevertheless, here or purpose is to localize a strange attractor, thus a specific and well known system representations are well known, so we will use this representations. Moreover from [15], the link between unidirectional synchronization and observation is well understood, so we implicitly refer to Arnold tongues concept [7] in order to localize the attractor. Finally, we used a multi observers technic [1] see figure 1 where the strange attractor localization is determined by a threshold on each residue. Indeed, for each sub-system we design an impulsive sub-observer which it converges to all statements. These sub-observer receives a sparse measurements, the error $\left(e_{1}=y-\hat{y}^{i}\right), i=1, \ldots r$ where $r$ is the number of subobserver) between the output of the strange attractor and each sub-observer allows us to detect the system that works.

\section{Simulation Results}

Let us consider the following switched chaotical dynamical systems:

1) Lorenz System:

$$
\Sigma_{\text {Lorenz }}=\left\{\begin{array}{l}
\dot{x}_{1}=13.5 x_{1}+10.5 x_{2}-x_{2} x_{3} \\
\dot{x}_{2}=22.5\left(x_{1}-x_{2}\right) \\
\dot{x}_{3}=-\frac{17}{6} x_{3}+x_{1} x_{2}
\end{array}\right.
$$




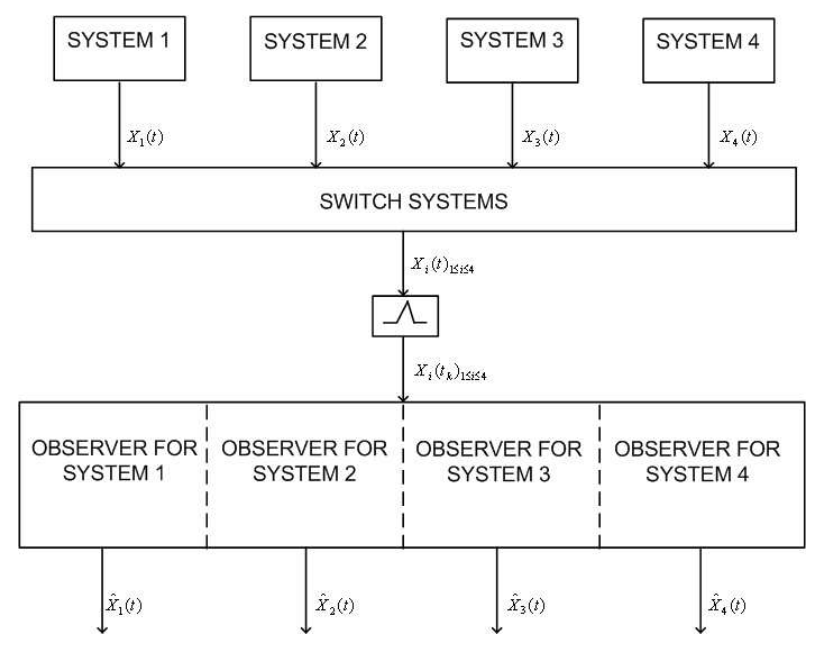

Fig. 1. Block diagram of multi-observer

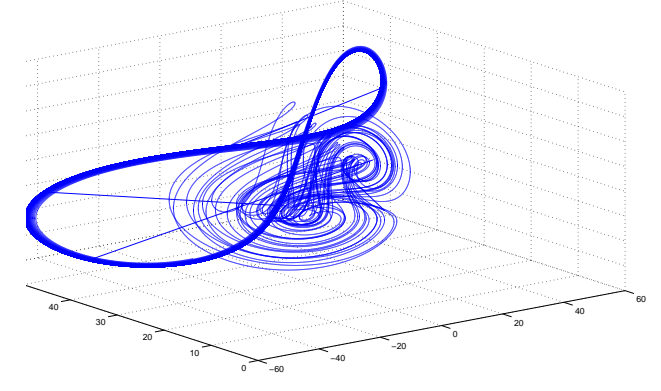

Fig. 2. Phases Portrait for Strange attractor

2) Lu system:

$$
\Sigma_{L u}=\left\{\begin{array}{l}
\dot{x}_{1}=22.2 x_{1}-x_{2} x_{3} \\
\dot{x}_{2}=30\left(x_{1}-x_{2}\right) \\
\dot{x}_{3}=-\frac{8.8}{3} x_{3}+x_{1} x_{2}
\end{array}\right.
$$

3) Chen system:

$$
\Sigma_{\text {Chen }}=\left\{\begin{array}{l}
\dot{x}_{1}=28 x_{1}+7 x_{2}-x_{2} x_{3} \\
\dot{x}_{2}=35\left(x_{1}-x_{2}\right) \\
\dot{x}_{3}=-3 x_{3}+x_{1} x_{2}
\end{array}\right.
$$

4) Qi system:

$$
\Sigma_{Q i}=\left\{\begin{array}{l}
\dot{x}_{1}=24\left(x_{1}+x_{2}\right)-x_{2} x_{3} \\
\dot{x}_{2}=42.5\left(x_{1}-x_{2}\right)+x_{1} x_{3} \\
\dot{x}_{3}=-13 x_{3}+x_{1} x_{2}
\end{array}\right.
$$

where $\left(x_{1}, x_{2}, x_{3}\right) \in \mathbb{R}^{3}$ and $y\left(t_{k}\right)=x_{1}\left(t_{k}\right)$ with $t_{k} \in$ $[0.1,0.4]$ is the measurement state. The hybrid behavior of the considered system is represented by its phase portrait in figure 2, this figure shows a trajectory simulation $x$ of the switched chaotic system. These chaotic subsystems switch in a random manner during a period $T_{r}>>t_{k}$, for $r, k>0$. In order to estimate the hybrid trajectory and detect the instant of switching, we propose a multi-observer which contains four sub-observers such that each sub-observer converges to the subsystem that corresponds, then we obtain:

1) Lorenz observer:

$$
O B S_{\text {Lorenz }}=\left\{\begin{array}{l}
\dot{\hat{x}}_{1}=13.5 \hat{x}_{1}+10.5 \hat{x}_{2}-\hat{x}_{2} \hat{x}_{3} \\
\dot{\hat{x}}_{2}=22.5\left(\hat{x}_{1}-\hat{x}_{2}\right) \\
\dot{\hat{x}}_{3}=-\frac{17}{6} \hat{x}_{3}+\hat{x}_{1} \hat{x}_{2} \\
\hat{x}_{1}\left(t_{k}^{+}\right)=x_{1}\left(t_{k}\right)
\end{array}\right.
$$

The observation error $e_{i}=x_{i}-\hat{x}_{i}$ are described by

$$
E R_{\text {Lorenz }}=\left\{\begin{array}{l}
\dot{e}_{1}=13.5 e_{1}+10.5 e_{2}-\hat{x}_{2} e_{3}+x_{3} e_{2} \\
\dot{e}_{2}=22.5\left(e_{1}-e_{2}\right) \\
\dot{e}_{3}=-\frac{17}{6} e_{3}+\hat{x}_{1} e_{2}+x_{2} e_{1} \\
e_{1}\left(t_{k}^{+}\right)=0
\end{array}\right.
$$

where

$$
f_{1}\left(e_{1}, e_{2}, e_{3}\right)=13.5 e_{1}+10.5 e_{2}-\hat{x}_{2} e_{3}+x_{3} e_{2}
$$

and

$f_{2}\left(e_{1}, e_{2}, e_{3}\right)=\left(\begin{array}{cc}-22.5 & 0 \\ \hat{x}_{2} & -\frac{17}{3}\end{array}\right)\left(\begin{array}{c}e_{2} \\ e_{3}\end{array}\right)+\left(\begin{array}{c}22.5 \\ x_{2}\end{array}\right) e_{1}$

Since $\hat{x}_{2}$ and $x_{3}$ are bounded, then the function $f_{1}$ is uniformly Lipschitz. Setting $E_{2}=\left(\begin{array}{c}e_{2} \\ e_{3}\end{array}\right)$, We use $V_{2}\left(e_{2}, e_{3}\right)=$ $\left(\begin{array}{ll}e_{2} & e_{3}\end{array}\right)\left(\begin{array}{cc}p_{1} & 0 \\ 0 & p_{2}\end{array}\right)\left(\begin{array}{l}e_{2} \\ e_{3}\end{array}\right)$ Lyapunov function with $p_{1}, p_{2}>0$, we obtain

$$
\begin{aligned}
& \frac{\partial V_{2}}{\partial E} A_{2} E_{2} \\
= & \left(\begin{array}{c}
e_{2} \\
e_{3}
\end{array}\right)^{T}\left(\begin{array}{cc}
-45 p_{1} & \hat{x_{2}} p_{2} \\
\hat{x_{2}} p_{2} & -\frac{35}{3} p_{2}
\end{array}\right)\left(\begin{array}{c}
e_{2} \\
e_{3}
\end{array}\right) \\
\leq & \lambda_{\min }\left(\begin{array}{cc}
-45 p_{1} & \hat{x_{2}} p_{2} \\
\hat{x_{2}} p_{2} & -\frac{35}{3} p_{2}
\end{array}\right)\left\|E_{2}\right\|_{2}^{2}
\end{aligned}
$$

then we can choose $p_{1}$ and $p_{2}$, such that $\lambda_{\min }\left(\begin{array}{cc}-45 p_{1} & \hat{x_{2}} p_{2} \\ \hat{x_{2}} p_{2} & -\frac{35}{3} p_{2}\end{array}\right)<0$ Hence, all the conditions of theorem 2 are satisfied, and we conclude that the Observer (17) converge to the Lorenz system (13).

2) Lu observer:

$$
O B S_{L u}=\left\{\begin{array}{l}
\dot{\hat{x}}_{1}=22.2 \hat{x}_{1}-\hat{x}_{2} \hat{x}_{3} \\
\hat{\hat{x}}_{2}=30\left(\hat{x}_{1}-\hat{x}_{2}\right) \\
\dot{\hat{x}}_{3}=-\frac{8.8}{3} \hat{x}_{3}+\hat{x}_{1} \hat{x}_{2} \\
\hat{x}_{1}\left(t_{k}^{+}\right)=x_{1}\left(t_{k}\right)
\end{array}\right.
$$

The observation error is described by

$$
E R_{L u}=\left\{\begin{array}{l}
\dot{e}_{1}=22.2 e_{1}-\hat{x}_{2} e_{3}-x_{3} e_{2} \\
\dot{e}_{2}=30\left(e_{1}-e_{2}\right) \\
\dot{e}_{3}=-\frac{8.8}{3} e_{3}+\hat{x}_{1} e_{2}+x_{2} e_{1} \\
e_{1}\left(t_{k}^{+}\right)=0
\end{array}\right.
$$

where

$$
f_{1}\left(e_{1}, e_{2}, e_{3}\right)=22.2 e_{1}-\hat{x}_{2} e_{3}+x_{3} e_{2}
$$


and

$$
f_{2}\left(e_{1}, e_{2}, e_{3}\right)=\left(\begin{array}{cc}
30 & 0 \\
\hat{x}_{2} & -\frac{8.8}{3}
\end{array}\right)\left(\begin{array}{l}
e_{2} \\
e_{3}
\end{array}\right)+\left(\begin{array}{l}
30 \\
x_{2}
\end{array}\right) e_{1}
$$

with the same argument of the first system (Lorenz system), we conclude that the observer (19) converge to the Lu system (14).

3) Chen observer:

$$
O B S_{\text {Chen }}=\left\{\begin{array}{l}
\dot{\hat{x}}_{1}=28 \hat{x}_{1}+7 \hat{x}_{2}-\hat{x}_{2} \hat{x}_{3} \\
\dot{\hat{x}}_{2}=35\left(\hat{x}_{1}-\hat{x}_{2}\right) \\
\dot{\hat{x}}_{3}=-3 \hat{x}_{3}+\hat{x}_{1} \hat{x}_{2} \\
\hat{x}_{1}\left(t_{k}^{+}\right)=x_{1}\left(t_{k}\right)
\end{array}\right.
$$

The observation error is described by

$$
E R_{\text {Chen }}=\left\{\begin{array}{l}
\dot{e}_{1}=28 e_{1}+7 e_{2}-\hat{x}_{2} e_{3}-x_{3} e_{2} \\
\dot{e}_{2}=35\left(e_{1}-e_{2}\right) \\
\dot{e}_{3}=-3 e_{3}+\hat{x}_{1} e_{2}+x_{2} e_{1} \\
e_{1}\left(t_{k}^{+}\right)=0
\end{array}\right.
$$

where

$$
f_{1}\left(e_{1}, e_{2}, e_{3}\right)=28 e_{1}+7 e_{2}-\hat{x}_{2} e_{3}+x_{3} e_{2}
$$

and

$$
f_{2}\left(e_{1}, e_{2}, e_{3}\right)=\left(\begin{array}{cc}
35 & 0 \\
\hat{x}_{2} & -3
\end{array}\right)\left(\begin{array}{l}
e_{2} \\
e_{3}
\end{array}\right)+\left(\begin{array}{l}
35 \\
x_{2}
\end{array}\right) e_{1}
$$

with the same argument of the first system (Lorenz system), we conclude that the observer (21) converge to the Lu system (15).

4) Qi observer:

$$
O B S_{Q i}=\left\{\begin{array}{l}
\dot{\hat{x}}_{1}=24\left(\hat{x}_{2}+\hat{x}_{1}\right)-\hat{x}_{2} \hat{x}_{3} \\
\dot{\hat{x}}_{2}=42.5\left(\hat{x}_{1}-\hat{x}_{2}\right)+\hat{x}_{1} \hat{x}_{3} \\
\dot{\hat{x}}_{3}=-13 \hat{x}_{3}+\hat{x}_{1} \hat{x}_{2} \\
\hat{x}_{1}\left(t_{k}^{+}\right)=x_{1}\left(t_{k}\right)
\end{array}\right.
$$

The observation error is described by

$$
E R_{Q i}=\left\{\begin{array}{l}
\dot{e}_{1}=24\left(e_{2}+e_{1}\right)-\hat{x}_{2} e_{3}-x_{3} e_{2} \\
\dot{e}_{2}=42.5\left(e_{1}-e_{2}\right)+\hat{x}_{1} e_{3}+x_{3} e_{1} \\
\dot{e}_{3}=-13 e_{3}+\hat{x}_{1} e_{2}+x_{2} e_{1} \\
e_{1}\left(t_{k}^{+}\right)=0
\end{array}\right.
$$

where

$$
f_{1}\left(e_{1}, e_{2}, e_{3}\right)=24 e_{1}+24 e_{2}-\hat{x}_{2} e_{3}-x_{3} e_{2}
$$

and

$$
\begin{aligned}
f_{2}\left(e_{1}, e_{2}, e_{3}\right) & =\left(\begin{array}{cc}
-42.5 & \hat{x_{1}} \\
\hat{x}_{1} & -13
\end{array}\right)\left(\begin{array}{l}
e_{2} \\
e_{3}
\end{array}\right) \\
& +\left(\begin{array}{c}
42.5+x_{3} \\
x_{2}
\end{array}\right) e_{1}
\end{aligned}
$$

$\mathrm{x}$ with the same argument of the first system (Lorenz system), we conclude that the observer (23) converge to the Lu system (16).

Figure 3 highlights the efficiency of the proposed method and shows respectively the convergence of each observer to his systems. the figure shows the corresponding switching trajectory. Also, if the observation error is zero for a subsystem, then we can confirm that it is one that works and vice versa. For example during the period $[0,10]$ we have $e_{1(Q I)}(t)=0$, then the system that works is the QI system For example during the period $[90,100]$ we have $e_{1 \text { (Lorenz) }}(t)=0$, then the system that works is the Lorenz system.

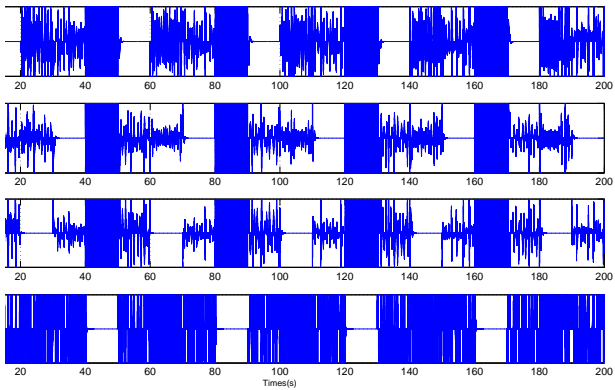

Fig. 3. Observation error

\section{CONCLUSION}

In this paper, based on a tentative to recycle a signal processing way to control system theory, an observer based control under sparse measurement was proposed. It is nothing to say that this study is a preliminary study and many researches must be investigated as for examples the link between RIP and Observability for system under sampling or again the generalization of the proposed design to nonlinear case.

\section{REFERENCES}

[1] B. Anderson, T. Brinsmead, F. De Bruyne, J. Hespanha, D. Liberzon, and A. Morse, "Multiple model adaptive control. i. finite controller coverings," George Zames Special Issue, International Journal of Robust and Nonlinear Control, vol. 10, pp. 909-929, 2000.

[2] L. Berec, "A multi-model method to fault detection and diagnosis: Bayesian solution. an introductory treatise," International Journal of Adaptive Control and Signal Processing, vol. 12, p. 8192, 1998.

[3] A. Bhagwat, R. Srinivasan, and P. Krishnaswamy, "Multi-linear modelbased fault detection during process transitions," Chemical Engineering Science, vol. 58, p. 1649 1670, 2003.

[4] S. Bhattachara and T. Basar, "Sparcity based feedback design: a new paradigm in opportunistic sensing," IEEE ACC, 2011

[5] E. Candès and M. B. Walkin, "An introduction to compressive sampling," IEEE Signal Processing Magazine, vol. 21, no. 2, pp. 21-30, 2008.

[6] A. Chaillet and A. Lorìa, "Uniform semiglobal practical asymptotic stability for non-autonomous cascaded systems and applications," $\mathrm{Au}$ tomatica, vol. 44, pp. 337-347, 2008.

[7] G. Chen and X. Dong, "From chaos to ,order," World Scientific, 1998.

[8] D. Donoho, I. Drori, V. Stoden, Y. Tsaig, and M. Shahram, "Sparselab," http://sparselab.stanford.edu.

[9] J.-P. Gauthier and I. Kupka, "Deterministic observation theory and applications," Cambridge University Press, 2001.

[10] S. Hamaci and A. Benfkir, "Optimization of resources for a behavior controlled of petri nets with multipliers in dioid algebra," in EURO2010. 24th European Conference on Operational Research, Lisbon-Portugal, july 11-14 2010 .

[11] W. Kang, "Bifurcation and normal form of nonlinear control systemspart 1 \& part 2," SIAM J. Control and Optimization, vol. 36, pp. 193-212 \& 213-232, 1998

[12] W. Kang and A. J. Krener, "Extended quadratic controller normal form and dynamic state feedback linearization of nonlinear systems," SIAM J. Control and Optimization, vol. 30, pp. 1319-1337, 1992.

[13] Y. Khaled, J.-P. Barbot, D. Benmerzouk, and K. Busawon, "A new type of impulsive observer for hyperchaotic system," in IFAC 3th Conference on analysis and control of chaotic systems, Cancum, Mxico, June 20 th-22th 20122012.

[14] A. Krener and W. Respondek, "Nonlinear observer with linearizable error dynamics," SIAM J. Control and Optimization, pp. 197-216, 1985.

[15] H. Nijmeijer and I. Mareels, "An observer looks at synchronization," IEEE Transactions on Circuits and Systems-1: Fundamental theory and Applications, vol. 44, no. 10, pp. 882-891, 1997. 
[16] H. Poincar, "Solutions priodiques dans le voisinage d.un point d.quilibrelunes sans quadrature," Les Mthodes Nouvelles de la Mcanique Cleste, Gauthier-Villars, pp. 156-161, 1892.

[17] I. A. Tall and W. Respondek, "Feedback classification of nonlinear single-input control systems with controllable linearization: Normal forms, canonical forms, and invariants," SIAM Journal on Control and Optimization, vol. 41, no. 5, pp. 1498-1531, 2003.

[18] A. Woiselle, J. Starck, and M. Fadili, "3d data denoising and inpainting with the fast curvelet transform," J. of Mathematical Imaging and Vision (JMIV), vol. 39, no. 2, pp. 121-139, 2011.

[19] X. Xia and W. Gao, "Nonlinear observer design by observer error linearization," SIAM J. Control and Optimization, pp. 199-216, 1989.

[20] A. Yang, M. Gastpar, R. Bajcsy, and S. Sastry, "Distributed sensor perception via sparce representation," Proceddings of IEEE, vol. 98, no. 6, pp. 1077-1088, 2010.

[21] L. Yu, H. Sun, J. P. Barbot, and G. Zheng, "Bayesian compressive sensing for cluster structured sparse signals," Signal Processing, vol. 92, pp. 259-269, 2012.

[22] G. Zheng, D. Boutat, and J. P. Barbot, "Single output-dependent observability normal form," SIAM, J. Control Optim, vol. 46, pp. 2242-2255, 2007. 